\title{
Enhancer of Zeste Homolog 2 (Ezh2) is essential for patterning of multiple musculoskeletal tissues but dispensable for tendon differentiation
}

Deepanwita Pal ${ }^{1}$, Scott M. Riester², Bashar Hasan², Sara F. Tufa' ${ }^{1}$, Amel Dudakovic², Douglas R. Keene ${ }^{1,3}$, Andre J. van Wijnen ${ }^{2, \#}$ and Ronen Schweitzer ${ }^{1,3, \#}$

${ }^{1}$ Research Division, Shriners Hospital for Children, Portland, Oregon, United States of America

2 Department of Orthopedic Surgery, Mayo Clinic College of Medicine, Mayo Clinic, Rochester, Minnesota; Biochemistry \& Molecular Biology, Mayo Clinic College of Medicine, Mayo Clinic, Rochester, Minnesota; Center for Regenerative Medicine, Mayo Clinic, Rochester, Minnesota, United States of America

${ }^{3}$ Department of Orthopedics, Oregon Health \& Science University, Portland, United States of America

\section{\#Corresponding authors}

schweitz@ohsu.edu (RS)

vanwijnen.andre@mayo.edu (AvW) 


\section{Abstract}

An efficient musculoskeletal system depends on the precise assembly and coordinated growth and function of muscles, skeleton and tendons. However, the mechanisms that drive integrated musculoskeletal development and coordinated growth and differentiation of each of these tissues are still being uncovered. Epigenetic modifiers have emerged as critical regulators of cell fate differentiation, but so far almost nothing is known about their roles in tendon biology. Previous studies have shown that epigenetic modifications driven by Enhancer of zeste homolog 2 (EZH2), a major histone methyltransferase, have significant roles in vertebrate development including skeletal patterning and bone formation. We now find that targeting Ezh2 through the limb mesenchyme also has significant effects on tendon and muscle patterning, likely reflecting the essential roles of early mesenchymal cues mediated by Ezh2 for coordinated patterning and development of all tissues of the musculoskeletal system. Conversely, loss of Ezh2 in the tendon cells did not disrupt the tendon cell fate suggesting that tenocyte differentiation and tendon maturation are independent of Ezh2 signaling. 


\section{Introduction}

Epigenetic regulation comprises of heritable changes in gene expression that do not alter the underlying DNA sequence but instead rely on adapting the chromatin (1). Predominant epigenetic mechanisms include DNA methylation in CpG-rich promoters, non-coding RNAs and covalent modifications on specific residues in histones such as acetylation and methylation among others (2). Histone methylation is widely accepted as a key component of the epigenetic machinery that contributes to both stability and reversibility of gene expression (3) and has been associated with development and homeostasis (4). While histone methylation occurs on all basic residues, lysine methylations are the most extensively characterized (1). Methylation enzymes catalyze the addition of methyl groups donated from S-adenosylmethionine to histones and comprise of three families - (i) Su(var)3-9, Enhancer of Zeste, Trithorax (SET) - domain containing proteins, (ii) DOT1 (disruptor of telomeric silencing) - like proteins (iii) arginine Nmethyltransferase (PRMT) proteins. Although originally thought to be permanent modifications, the discovery of lysine-specific demethylase $1 \mathrm{~A}$ revealed the reversibility of histone methylation (5). The dynamic nature and reversibility of histone modifiers represents tremendous potential for therapeutic approaches and drug discovery, making them attractive targets for pharmacotherapies including orthopedic treatments $(6,7)$.

Breakthrough discoveries over the last decade have transformed our knowledge of epigenetic mechanisms in development and disease [8]. However, analysis of the function of epigenetic regulators in musculoskeletal biology is still evolving. In this work, we focused on a major histone methyltransferase called Enhancer of zeste homolog 2 (EZH2), a mammalian homolog of the Drosophila protein $E(Z)$ (originally termed Enx-1) (8). Ezh2 is the catalytic subunit of the Polycomb repressive complex (PRC2) and represents a highly evolutionarily conserved Polycomb group (PcG) member [10]. The functional relevance of the PcG proteins is highlighted 
by the numerous developmental defects reported in mice deficient for these proteins (9-11). Ezh2 is distinct among the PcG proteins due to its evolutionarily conserved SET domain, which catalyzes histone H3 lysine (K) 27 methylation, eventually leading to transcriptional repression. Ezh2 is important for early vertebrate development, as is evident from the phenotype of mice lacking Ezh2, which die around gastrulation (12). In zebrafish, Ezh2 activity is dispensable for tissue specification but required for the maintenance of differentiated cell fates in the heart, liver and pancreas (13). Ezh2 is also required for neural crest-derived bone and cartilage formation, with severe craniofacial defects observed upon Ezh2 depletion in neural crest cells (14). In limb development, Ezh2 regulates anteroposterior patterning via maintenance of Hox gene expression (15). Loss of Ezh2 in uncommitted limb mesenchymal cells leads to skeletal malformations and defective bone formation, reflecting key roles for Ezh2 in osteoblast maturation and skeletal development (16-18). Because of the epigenetic role of Ezh2 in several principal stages of development, it is likely that it has essential roles in the development of other musculoskeletal tissues, but this hypothesis has not yet been experimentally validated.

The musculoskeletal system is a coordinated assembly of the skeleton, muscles and tendons, where the tendons transmit the force of muscle contraction to the skeleton thereby enabling joint movement. We recently established a model of musculoskeletal integration in the developing embryo (19) using expression of Scleraxis (Scx), a transcription factor, which serves as a distinctive marker for tendon cells from progenitor stages through development (20). Our work demonstrated the considerable interdependence of musculoskeletal tissues for coordinated growth and tissue formation. Considering the significant role of Ezh2 in cell fate determination for various tissues including osteogenic differentiation $(17,18)$, we hypothesized that it may also play a role in cell fate determination of muscles and tendons. The goal of this study was to test if tendons are affected by the epigenetic signaling of Ezh2 and whether Ezh2 is involved in tenogenic differentiation. 
We find that loss of Ezh2 in limb mesenchyme resulted in dramatic changes to muscle and tendon pattern likely reflecting early mesenchymal signals required for patterning and coordinated growth of the musculoskeletal system. Even though Ezh2 is required for tendon patterning, we find that the regulation of tenocyte differentiation and maturation does not depend on Ezh2 signaling.

\section{Materials and methods}

\section{Mice Breeding Scheme and Animal Welfare}

Existing mouse lines used in these studies were described previously: Ezh2 ${ }^{\mathrm{fff}}(21), \operatorname{Pr} x 1^{\mathrm{Cre}}(22)$, ScxGFP tendon reporter (23). Direct targeting of Ezh2, to determine tendon specific effects, was performed using the tendon specific deletor Scx Cre (24). All animal procedures were approved by the Institutional Animal Care and Use Committee at the Oregon Health \& Science University and the Mayo Clinic and are consistent with animal care guidelines.

\section{X-ray imaging}

To measure differences in skeletal development, X-ray imaging was employed to assess gross anatomical structure at 3-4 weeks. Both males and females were analyzed to account for gender specific effects.

\section{Skeletal preparations}

Cartilage and bones of the forelimbs harvested from mice were visualized after staining with Alcian blue (Sigma) and Alizarin red S (Sigma) and subsequent clarification of soft tissue with $\mathrm{KOH}(25)$.

\section{Tissue Harvest}


Mice were euthanized by carbon dioxide asphyxiation followed by thoracotomy. Forelimbs were resected as follows: a small slit in the skin near the scapula was made. Then the skin was lifted using forceps and a slit was made through the length of the limb on the dorsal side. This superficial slit through the skin was made up to the metacarpal-phalangeal joint in the digits, to enable better fixation of the tissues inside.

\section{Histology}

For whole-mount visualization, tissues were skinned and images were captured on an MZFLIII dissecting microscope (Leica) with an EOS50D camera (Canon). Patterning of limb tendons and muscles was acquired in serial transverse sections of $12 \mu \mathrm{m}(26)$ and immunostaining was performed as previously described (27). Tendons were visualized using ScxGFP expression or anti-collagen I (Coll) antibody (Southern Biotech). Antigen retrieval using citrate buffer for the Coll staining was performed using the PELCO Biowave microwave (Ted Pella, Inc). A monoclonal antibody for My32 (Sigma Aldrich) was used to detect muscle-specific Type II myosin heavy chain ( $\mathrm{MHC}$ ). Images were captured using Zeiss ApoTome2 on Axiolmager (Zeiss). Image processing was carried out using Zeiss Zen software.

\section{Transmission electron microscopy (TEM)}

P21 and P28 mouse limbs were skinned and fixed in 1.5\% glutaraldehyde/1.5\% paraformaldehyde (Tousimis Research Corporation) in Dulbecco's serum-free media (DMEM) containing $0.05 \%$ tannic acid at $4^{\circ} \mathrm{C}$ for $1-2$ weeks. Forelimbs were then rinsed in DMEM followed by extensive decalcification in $0.2 \mathrm{M}$ EDTA in 50mM Tris in a Pelco 3450 microwave (Ted Pella, Inc) at $94.5 \mathrm{~W}$. Tissues were fixed again in $1.5 \%$ glutaraldehyde/1.5\% paraformaldehyde with $0.05 \%$ tannic acid over night at $4^{\circ} \mathrm{C}$, rinsed in DMEM, then post-fixated in $1 \%$ OsO4 overnight. Samples were washed in DMEM, dehydrated in a graded series of ethanol to $100 \%$, rinsed in propylene oxide, and finally embedded in Spurrs epoxy. Tendons of 
bioRxiv preprint doi: https://doi.org/10.1101/2020.10.30.361949; this version posted October 30, 2020. The copyright holder for this preprint (which was not certified by peer review) is the author/funder, who has granted bioRxiv a license to display the preprint in perpetuity. It is made available under aCC-BY 4.0 International license.

interest were identified by collecting $1 \mu \mathrm{m}$ sections stained with an epoxy tissue stain (Electron Microscopy Sciences). Ultrathin sections containing tendons of interest were cut at $80 \mathrm{~nm}$, contrasted with uranyl acetate and lead citrate, and viewed with an FEI G20 TEM operated at $120 \mathrm{kV}$ using an AMT $2 \times 2 \mathrm{~K}$ camera. TEM images of transverse sections were collected at several magnifications to enable morphological visualization of the collagen fibrils and gross tendon appearance. 


\section{Results}

\section{Deletion of Ezh2 in limb mesenchyme affects tendon and musculoskeletal patterning}

It was previously reported that global knockout of Ezh2 in mice results in embryonic lethality (12) precluding analysis of specific roles in tendon development. Hence, for initial analysis, we targeted Ezh2 in limb mesenchyme using Prx1Cre (22). Previous studies established a role for Ezh2 in anterior posterior patterning of the limb (15) and associated loss of Ezh2 with several skeletal abnormalities including decreased vertebral height, shortened limbs and prematurely fused cranial structures $(15,16,28)$. However, the effects of the loss of Ezh2 on patterning and differentiation of the soft musculoskeletal tissues were not examined in these reports. Consistent with previous studies, we found that Ezh2Prx1Cre mutant limbs were considerably shorter compared to WT littermates and displayed anteroposterior patterning defects (15) (Fig 1). Ezh2 ${ }^{\text {Prx1Cre }}$ mutants also displayed movement limitations likely reflecting broad disruptions to the musculoskeletal system in addition to the reported skeletal defects (data not shown).

Fig 1. Deletion of Ezh2 in the limb mesenchyme affects skeletal patterning.

Whole mount images of WT and Ezh2 ${ }^{\text {Prx } 1 \text { re }}$ limbs at 3 weeks

To examine the effects of targeting Ezh2 in limb mesenchyme on the soft musculoskeletal tissues, the tendons and muscles, we stained for Collagen I (Coll; encoded by Col1a1 or Col1a2) and Myosin Heavy Chain (MHC; encoded by Myh1) respectively (Fig 2B). The patterning of both the tendons and muscles was substantially affected in mutant limbs. Paw movement is controlled by two broad categories of muscles, intrinsic muscles whose muscle bellies are found within the paw and extrinsic muscles with muscle bellies that reside in the arm and long tendons that connect these muscles to their specific insertion points in the paw $(26$, 29). 
Fig 2. Deletion of Ezh2 in the limb mesenchyme affects muscle and tendon patterning.

A. Schematic of the ventral side of the forelimb that illustrates the positions of the limb sections on the panels on the right. B. Images of transverse sections at the metacarpals (L1) and the wrist (L2) of WT and Ezh2 ${ }^{\text {Prx1Cre }}$ mutant limbs at P21. Coll staining was used to visualize tendons in WT and mutant limbs respectively while My32 staining was used for muscle. Yellow, pink, orange and teal arrowheads highlight the flexor digitorium profundus, flexor digitorium superficialis, extensor digitorium communis and the lateral extensor tendons respectively. White arrows indicate interosseous tendons. I, L, T and HT indicate the interosseous, lumbricals, thenar and hypothenar muscles respectively.

All extensor muscles are extrinsic and their tendons were severely disrupted in mutant forelimbs. Extensor communis tendons extend through the length of the digits and the skeletal insertions for other extensors are found in various carpal or metacarpal bones (26). Only two communis extensor tendons were detected in mutant limbs (Fig 2B, orange arrowheads) and most of the other lateral extensor tendons were missing in the mutant (Fig 2B, teal arrowheads). The major flexor muscles are the flexor digitorium profundus (FDP) extrinsic muscles. The FDP tendons are fused in the wrist and carpal regions and separate to individual tendons that insert in the distal part of each digit $(26,29)$. These FDP tendons, the most robust paw tendons, were significantly smaller throughout the paw of mutant forelimbs (Fig 2B, yellow arrowheads). The two major intrinsic groups of paw muscles are the interosseous muscles located directly ventral to the metacarpal bones and the lumbrical muscles that extend through the length of the metacarpals directly between FDP tendons ((26) and Fig 2B). The interosseous muscles were also significantly smaller and mis-patterned in mutant limbs (Fig 2B, I). Moreover, interosseous tendons could not be detected in distal sections and only a small subset of these tendons were detected more proximally (Fig 2B, white arrows). Mutant effects were even more dramatic on 
the lumbrical muscles. Lumbrical muscles have a distinct morphology between the FDP tendons in sections at metacarpal level ((26) and Fig $2 \mathrm{~B})$ and these muscles were completely absent in mutant paws (Fig 2B, L) .

The severe effects of the loss of Ezh2 on musculoskeletal patterning were further reflected in the fate of the flexor digitorium superficialis (FDS) muscles and tendons. While FDS muscles are extrinsic to the paw, they follow a unique developmental program. The FDS muscles first differentiate with multinucleated myofibers in the forepaw and subsequently translocate out of the paw and into the arm leaving behind the FDS tendons at their distal ends (30). The translocation of FDS muscles involves dramatic muscle elongation at the proximal end with concurrent retraction from the distal end and both of these processes did not occur in the Ezh2 Prx1Cre mutant. In mutant sections, we found muscles directly underneath the FDP tendons instead of the FDS tendons that usually occupy this space (Fig 2B, yellow asterisks). We previously identified such a configuration in the limbs of Scx and paralyzed mutants in which FDS muscle translocation failed (27).

Together, these results demonstrate that the loss of Ezh2 had a profound effect on the patterning, organization and size of muscles and tendons. Combined with previous reports of significant disruptions of skeletal patterning in Ezh2Prx1Cre mutants, these results suggest an essential role for Ezh2 in overall musculoskeletal patterning.

\section{Ezh2 is dispensable for the tendon cell fate and tenocyte maturation}

Ezh2 was implicated in cell fate differentiation and maturation and in addition to the early effects on skeletal development, Ezh2 has essential roles during osteogenesis (17). Since tendons were greatly affected in the Ezh2Prx1Cre mutant, we investigated whether Ezh2 is also involved in tenocyte differentiation and the regulation of tendon growth and maturation. To examine Ezh2 function directly in the tenocyte lineage, we used a tendon specific deletor Scx Cre (24) to target 
Ezh2 specifically in tenocytes. First, we wanted to determine if skeletal patterning or growth was affected in these mutants, to exclude the possibility of secondary effects of skeletal disruptions on the tendons. Contrary to the Ezh2Prx1Cre mutant, skeletal pattern was not affected in Ezh2 ${ }^{\text {ScxCre }}$ mutants as evident from whole mount images, skeletal preparations and X-ray images (Fig 3). Furthermore, using the ScxGFP tendon reporter (23), we found that in mutant limbs the tendon system was largely intact and there were no significant differences between four week old WT and Ezh2ScxCre mutant littermates in tendon patterning (Fig 4B and C).

Fig 3. Targeted deletion of Ezh2 in tendons did not result in defects in skeletal patterning. Whole mount images (A) and skeletal preparations (B) and X-ray images (C) of WT and Ezh2 ${ }^{\text {ScxCre }}$ limbs at 4 weeks.

Fig 4. Loss of Ezh2 in tendon lineage cells did not affect tendon patterning.

A. Schematic of the ventral side of the forelimb that illustrates the positions of the limb sections on the panels on the right. B. Images of transverse sections of WT and Ezh2 ${ }^{\text {ScxCre }}$ mutant at 4 weeks showing ScxGFP and Coll expression at the metacarpals (L1) and the wrist (L2). C. High magnification of the regions defined by the insets on the left showing transverse sections of the flexors of the WT and Ezh2 ${ }^{\text {ScxCre }}$ mutant.

Although we did not observe any tendon patterning defects in the $S c x^{\text {Cre }}$ mutant, we next examined the involvement of Ezh2 in tendon differentiation. The characteristic feature of a mature tendon is the highly organized and robust collagen matrix. The collagen matrix occupies most of the tendon volume and it is composed of an assembly of collagen fibrils with heterogeneous cross section area that extend in parallel arrays through the length of the tendon (31). The pattern and quantitative parameters of the collagen matrix are commonly examined in high magnification transmission electron microscope (TEM) images. To determine if the tendon 
cell fate was affected in Ezh2ScxCre mutants, we therefore analyzed the organization of the collagen matrix in these mutants. We find that the collagen matrix was not disrupted in the tendons of Ezh2ScxCre mutants and fibril organization, shape and the distribution of fibril diameters all appear similar in comparison of TEM images from section of mutant and WT littermate tendons (Fig 5A).

\section{Fig 5. Ezh2 is dispensable for tendon cell fate and tenocyte maturation}

A. TEM images of WT and Ezh2 $2^{S c x C r e}$ mutant showing collagen structure in transverse sections of flexor digitorium profundus tendon in the digits (L1) and extensor carpi ulnaris in the wrist (L2). Scale: $500 \mathrm{~nm}$, Magnification: 50,000X. B. TEM images of WT and Ezh2 ${ }^{\text {Prx1Cre }}$ mutant showing collagen structure in transverse sections through flexor digitorium profundus tendon in the digits (L1) and extensor carpi radialis brevis tendon in the wrist (L2). Scale: 500nm, Magnification: 50,000X.

The onset of $\operatorname{Prx} 1^{\text {Cre }}$ activity in early limb mesenchyme at E9.5 precedes recombination by the Scx ${ }^{\text {Cre }}$ driver that emerges in tendon cells around E13.5. Since the tendons and all other tissues of the musculoskeletal system were profoundly affected in Ezh2Prx1Cre mutants, we investigated whether early targeting of $\mathrm{EZH} 2$ in the Ezh2 $2^{\text {rx1Cre }}$ mutants had a more significant effect on the tendon cell fate. We therefore also analyzed the collagen matrix in tendons from these mutants. However, we found that despite the considerable disruption of tendon pattern and size in the Ezh2Prx1Cre mutants, the loss of Ezh2 had no effect on tendon maturation and differentiation and the collagen matrix in mutant tendon was similar to that of WT littermates (Fig 5B). Taken together, our results suggest that while Ezh2 activity is essential in early stages of limb development for tendon and overall musculoskeletal patterning, Ezh2 activity is dispensable within the tenocyte-lineage and thus not essential for the regulation of the tendon cell fate. 


\section{Discussion}

In this study, we have examined the role of the histone methyltransferase Ezh2 in tendon development. Our findings show that Ezh2-mediated signals are important for early tendon and overall musculoskeletal patterning, but Ezh2 is not essential for the regulation of tendon cell fate.

It was previously reported that Ezh2 plays an essential role in early limb patterning (15). The role of Ezh2 in very early aspects of limb patterning was further highlighted by the fact that anterior-posterior patterning was more dramatically affected when Ezh2 was targeted with $T^{\text {cre }}$ before the initiation of the limb bud compared with the outcome of targeting Ezh2 with Prx1Cre that becomes active only at the initial stages of limb bud formation (15). In agreement with this, we consistently found in Ezh2Prx1Cre, substantial disruption of skeletal patterning and loss of digit 1 ((16) and Fig 1).

The developmental regulatory signals that underlie the specific pattern of the cartilage condensations in the developing embryo remain obscure and even less is known about the initial cues that guide muscle or tendon patterning. However, skeletal patterning is independent of the presence of muscles and the basic layout of muscle pattern can be detected even in a skeletal-less limb $(19,24)$. Early patterning of all the musculoskeletal tissues may therefore be guided by signals from the limb mesenchyme $(32,33)$. It is not yet known what these signals are or if all musculoskeletal tissues are affected by the same signals or if there are distinct cues for each of these tissues. It was therefore interesting to examine the effects of the loss of Ezh2 on muscle and tendon patterning. Remarkably, the tendons and the muscles were also severely affected in the Ezh2 Prx1Cre mutant, suggesting that Ezh2 activity underlies basic aspects of 
mesenchymal patterning that guide the patterning and development of all musculoskeletal tissues.

The loss of Ezh2 in limb mesenchyme resulted in severe and complex effects on tendon development. Various extensor and flexor tendons were missing in mutant limbs and the existing tendons were drastically smaller that their WT counterparts, an effect seen most dramatically in the FDP tendons (Fig 2B). Contrary to the independent development of cartilage and muscles, tendon development is inherently dependent on the other musculoskeletal tissues $(19,24,34,35)$. We and others have previously demonstrated that tendon induction and development within the autopod is dependent on cartilage and tendon development in the zeugopod is dependent on the presence of muscles $(19,35)$. Because of the interdependence of tendon development in the paw on signals from the cartilage, the failure of tendon development may reflect an indirect effect through disruption of signal(s) that emanate from the cartilage to regulate tendon formation. On the other hand, the observed tendon defects may also reflect a direct role for Ezh2 in tendon formation by regulating specific mesenchymal cues that guide or instruct tendon development. It is not possible at this time to determine if Ezh2 activity plays a direct or indirect role in the development of these tendons.

The complex role of Ezh2 activity in regulation of muscle and tendon development is especially reflected in the effects on flexor muscles and tendon. FDP tendons were significantly smaller, FDS tendons were missing in the mutant paw and FDS muscles were found in the paw instead of their normal position in the arm. We previously described a unique developmental path for the FDS muscles and tendons (30). The FDS muscles first organize as fully formed muscles in the paw. They subsequently elongate proximally and translocate from the paw and into the arm. The FDS tendons are formed in the wake of the FDS muscles as they recede from the paw (30). In mutants that disrupt tendon development ( $S c x)$ or muscle activity (mdg) muscle translocation is disrupted and the FDS muscles remain within the paw, resulting also in failure of FDS tendon 
development $(19,27)$. The FDS muscle and tendon phenotype in Ezh2Prx1Cre mutant limbs may thus reflect a direct failure of muscle activity or tendon development. It may however also reflect the absence of other cues that initiate, support or propel FDS muscle movement.

Notably, failures in tendon growth could also reflect a direct role for Ezh2 in the regulation of tendon growth. However, the tendon phenotypes observed in Ezh2Prx1Cre mutant limbs were not recapitulated when Ezh2 was targeted with the tendon-specific deletor, Scx Cre, suggesting that Ezh2 is most likely essential for early patterning of all musculoskeletal tissues. However, we cannot rule out the possibility that Ezh2 has an early role in limb mesenchyme, which is later essential for tendon differentiation and growth. Since the onset of $S c x^{C r e}$ activity is later than that of $\operatorname{Prx} 1^{\text {Cre }}$, such an early function may be bypassed in the Ezh2ScxCre mutant. Finally, it is well established that tendon growth is dependent on biomechanical activity (19). Considering the skeletal patterning disruptions in Ezh2 ${ }^{\text {Prx1Cre }}$ mutants and the mobility issues they experience, it is conceivable that some aspects of the tendon phenotypes may be attributed to restricted biomechanical activity.

Ezh2 activity has been implicated in cell fate determination and differentiation (36), as well as maintenance of the stem cell state in the mesenchymal linages (37). The complexity of these functions of Ezh2 was uncovered in studies of the skeletal tissues. Loss of Ezh2 in the osteogenic lineage resulted in reduced bone formation, but conditional loss of Ezh2 in uncommitted mesenchymal cells yields skeletal patterning defects, including shortened forelimbs, craniosynostosis and clinodactyly (17). Conversely, loss of Ezh2 in chondrocytes did not disrupt cartilage development despite the appearance of osteogenic gene expression in the mutant chondrocytes (38). We therefore examined if Ezh2 has a role in tenogenic differentiation, but did not find any effect on the normal production of the prototypic extracellular matrix of tendons in mutant tendons (Fig 5). 
Finally, in analysis of the consequences of targeting the Ezh2 gene it is important to consider the possible roles of functional homologs that may substitute to provide the same molecular activity in different tissues or at different stages of development and maturation. Indeed, Ezh1, a functional homolog of Ezh2, is ubiquitously expressed whereas Ezh2 expression is associated mostly with proliferating tissues (39). Hence, it is possible that the homologs share redundant features that would allow Ezh1 to compensate for the functions of Ezh2 in various tissues including tendons. A recent report (40) indeed suggests that Ezh1 and Ezh2 could potentially compensate for each other in skeletal development. It remains to be seen if such a mechanism is also active during tendon development. 


\section{Acknowledgments}

We would like to thank all past and present members of our laboratories, including Brian Pryce, Fuhua Xu, Emily Camilleri and Roman Thaler for stimulating discussions, providing reagents and generously sharing ideas.

\section{Author contributions}

Conceptualization: Deepanwita Pal, Scott Riester, Amel Dudakovic, Andre van Wijnen, Ronen Schweitzer

Data curation: Deepanwita Pal

Formal analysis: Sara Tufa, Douglas Keene

Funding acquisition: Andre van Wijnen, Ronen Schweitzer

Investigation: Deepanwita Pal, Scott Riester, Bashar Hasan, Amel Dudakovic, Sara Tufa

Methodology: Deepanwita Pal, Scott Riester, Bashar Hasan, Amel Dudakovic, Sara Tufa, Douglas Keene, Andre van Wijnen, Ronen Schweitzer

Project administration: Andre van Wijnen, Ronen Schweitzer

Resources: Douglas Keene, Andre van Wijnen, Ronen Schweitzer

Supervision: Andre van Wijnen, Ronen Schweitzer

Validation: Deepanwita Pal, Scott Riester, Bashar Hasan, Amel Dudakovic, Andre van Wijnen, Ronen Schweitzer

Visualization: Deepanwita Pal, Amel Dudakovic, Andre van Wijnen, Ronen Schweitzer

Writing - Original draft: Deepanwita Pal, Ronen Schweitzer

Writing - Review and editing: Deepanwita Pal, Scott Riester, Bashar Hasan, Sara Tufa, Amel Dudakovic, Douglas Keene, Andre van Wijnen, Ronen Schweitzer

\section{Funding}


This work was supported by funding from the National Institute of Health (R01 AR067211 to RS and R01 AR069049 to AvW) and Shriners Hospitals for Children (SHC 85410-POR-16 to RS). We also thank William and Karen Eby for their generous philanthropic support.

\section{References}

1. Allis CD, Jenuwein $T$. The molecular hallmarks of epigenetic control. Nat Rev Genet. 2016;17(8):487-500.

2. Barrero MJ, Boue S, Izpisua Belmonte JC. Epigenetic mechanisms that regulate cell identity. Cell Stem Cell. 2010;7(5):565-70.

3. Martin C, Zhang Y. The diverse functions of histone lysine methylation. Nat Rev Mol Cell Biol. 2005;6(11):838-49.

4. Oppermann $U$. Why is epigenetics important in understanding the pathogenesis of inflammatory musculoskeletal diseases? Arthritis Res Ther. 2013;15(2):209.

5. Byvoet P, Shepherd GR, Hardin JM, Noland BJ. The distribution and turnover of labeled methyl groups in histone fractions of cultured mammalian cells. Arch Biochem Biophys. $1972 ; 148(2): 558-67$.

6. Arrowsmith $\mathrm{CH}$, Bountra C, Fish PV, Lee K, Schapira M. Epigenetic protein families: a new frontier for drug discovery. Nat Rev Drug Discov. 2012;11(5):384-400.

7. van Wijnen AJ, Westendorf JJ. Epigenetics as a New Frontier in Orthopedic Regenerative Medicine and Oncology. J Orthop Res. 2019;37(7):1465-74.

8. Hobert $\mathrm{O}$, Jallal $\mathrm{B}$, Ullrich $\mathrm{A}$. Interaction of Vav with ENX-1, a putative transcriptional regulator of homeobox gene expression. Mol Cell Biol. 1996;16(6):3066-73.

9. Gould A. Functions of mammalian Polycomb group and trithorax group related genes. Curr Opin Genet Dev. 1997;7(4):488-94. 
10. van der Lugt NM, Domen J, Linders K, van Roon M, Robanus-Maandag E, te Riele H, et al. Posterior transformation, neurological abnormalities, and severe hematopoietic defects in mice with a targeted deletion of the bmi-1 proto-oncogene. Genes Dev. 1994;8(7):757-69.

11. Dudakovic A, van Wijnen AJ. Epigenetic Control of Osteoblast Differentiation by Enhancer of Zeste Homolog 2 (EZH2). Current Molecular Biology Reports. 2017;3(2):94-106.

12. O'Carroll D, Erhardt S, Pagani M, Barton SC, Surani MA, Jenuwein T. The polycombgroup gene Ezh2 is required for early mouse development. Mol Cell Biol. 2001;21(13):4330-6.

13. San B, Chrispijn ND, Wittkopp N, van Heeringen SJ, Lagendijk AK, Aben M, et al. Normal formation of a vertebrate body plan and loss of tissue maintenance in the absence of ezh2. Sci Rep. 2016;6:24658.

14. Schwarz D, Varum S, Zemke M, Scholer A, Baggiolini A, Draganova K, et al. Ezh2 is required for neural crest-derived cartilage and bone formation. Development. 2014;141(4):86777.

15. Wyngaarden LA, Delgado-Olguin P, Su IH, Bruneau BG, Hopyan S. Ezh2 regulates anteroposterior axis specification and proximodistal axis elongation in the developing limb. Development. 2011;138(17):3759-67.

16. Dudakovic A, Camilleri ET, Xu F, Riester SM, McGee-Lawrence ME, Bradley EW, et al. Epigenetic Control of Skeletal Development by the Histone Methyltransferase Ezh2. J Biol Chem. 2015;290(46):27604-17.

17. Dudakovic A, Camilleri ET, Paradise CR, Samsonraj RM, Gluscevic M, Paggi CA, et al. Enhancer of zeste homolog 2 (Ezh2) controls bone formation and cell cycle progression during osteogenesis in mice. J Biol Chem. 2018;293(33):12894-907.

18. Dudakovic A, Samsonraj RM, Paradise CR, Galeano-Garces C, Mol MO, GaleanoGarces D, et al. Inhibition of the epigenetic suppressor EZH2 primes osteogenic differentiation mediated by BMP2. J Biol Chem. 2020;295(23):7877-93. 
19. Huang AH, Riordan TJ, Pryce B, Weibel JL, Watson SS, Long F, et al. Musculoskeletal integration at the wrist underlies the modular development of limb tendons. Development. 2015;142(14):2431-41.

20. Schweitzer R, Chyung JH, Murtaugh LC, Brent AE, Rosen V, Olson EN, et al. Analysis of the tendon cell fate using Scleraxis, a specific marker for tendons and ligaments. Development. 2001;128(19):3855-66.

21. Su IH, Basavaraj A, Krutchinsky AN, Hobert O, Ullrich A, Chait BT, et al. Ezh2 controls B cell development through histone $\mathrm{H} 3$ methylation and Igh rearrangement. Nat Immunol. 2003;4(2):124-31.

22. Logan M, Martin JF, Nagy A, Lobe C, Olson EN, Tabin CJ. Expression of Cre Recombinase in the developing mouse limb bud driven by a Prxl enhancer. Genesis. $2002 ; 33(2): 77-80$.

23. Pryce BA, Brent AE, Murchison ND, Tabin CJ, Schweitzer R. Generation of transgenic tendon reporters, ScxGFP and ScxAP, using regulatory elements of the scleraxis gene. Dev Dyn. 2007;236(6):1677-82.

24. Blitz E, Viukov S, Sharir A, Shwartz Y, Galloway JL, Pryce BA, et al. Bone ridge patterning during musculoskeletal assembly is mediated through SCX regulation of Bmp4 at the tendon-skeleton junction. Dev Cell. 2009;17(6):861-73.

25. McLeod MJ. Differential staining of cartilage and bone in whole mouse fetuses by alcian blue and alizarin red S. Teratology. 1980;22(3):299-301.

26. Watson SS, Riordan TJ, Pryce BA, Schweitzer R. Tendons and muscles of the mouse forelimb during embryonic development. Dev Dyn. 2009;238(3):693-700.

27. Murchison ND, Price BA, Conner DA, Keene DR, Olson EN, Tabin CJ, et al. Regulation of tendon differentiation by scleraxis distinguishes force-transmitting tendons from muscleanchoring tendons. Development. 2007;134(14):2697-708. 
28. Hemming S, Cakouros D, Codrington J, Vandyke K, Arthur A, Zannettino A, et al. EZH2 deletion in early mesenchyme compromises postnatal bone microarchitecture and structural integrity and accelerates remodeling. FASEB J. 2017;31(3):1011-27.

29. Delaurier A, Burton N, Bennett M, Baldock R, Davidson D, Mohun TJ, et al. The Mouse Limb Anatomy Atlas: an interactive 3D tool for studying embryonic limb patterning. BMC Dev Biol. 2008;8:83.

30. Huang AH, Riordan TJ, Wang L, Eyal S, Zelzer E, Brigande JV, et al. Repositioning forelimb superficialis muscles: tendon attachment and muscle activity enable active relocation of functional myofibers. Dev Cell. 2013;26(5):544-51.

31. Benjamin M, Ralphs JR. Tendons and ligaments--an overview. Histol Histopathol. $1997 ; 12(4): 1135-44$.

32. Hasson P, DeLaurier A, Bennett M, Grigorieva E, Naiche LA, Papaioannou VE, et al. Tbx4 and tbx5 acting in connective tissue are required for limb muscle and tendon patterning. Dev Cell. 2010;18(1):148-56.

33. Colasanto MP, Eyal S, Mohassel P, Bamshad M, Bonnemann CG, Zelzer E, et al. Development of a subset of forelimb muscles and their attachment sites requires the ulnarmammary syndrome gene Tbx3. Dis Model Mech. 2016;9(11):1257-69.

34. Edom-Vovard F, Duprez D. Signals regulating tendon formation during chick embryonic development. Dev Dyn. 2004;229(3):449-57.

35. Kardon G. Muscle and tendon morphogenesis in the avian hind limb. Development. $1998 ; 125(20): 4019-32$.

36. Chou $\mathrm{RH}, \mathrm{Yu} \mathrm{YL}$, Hung MC. The roles of $\mathrm{EZH} 2$ in cell lineage commitment. Am J Transl Res. $2011 ; 3(3): 243-50$.

37. Sen B, Paradise CR, Xie Z, Sankaran J, Uzer G, Styner M, et al. beta-Catenin Preserves the Stem State of Murine Bone Marrow Stromal Cells Through Activation of EZH2. J Bone Miner Res. 2020;35(6):1149-62. 
38. Camilleri ET, Dudakovic A, Riester SM, Galeano-Garces C, Paradise CR, Bradley EW, et al. Loss of histone methyltransferase Ezh2 stimulates an osteogenic transcriptional program in chondrocytes but does not affect cartilage development. J Biol Chem. 2018;293(49):1900111.

39. Margueron R, Li G, Sarma K, Blais A, Zavadil J, Woodcock CL, et al. Ezh1 and Ezh2 maintain repressive chromatin through different mechanisms. Mol Cell. 2008;32(4):503-18.

40. Lui JC, Garrison P, Nguyen Q, Ad M, Keembiyehetty C, Chen W, et al. EZH1 and EZH2 promote skeletal growth by repressing inhibitors of chondrocyte proliferation and hypertrophy. Nat Commun. 2016;7:13685. 


\section{Ezh2 $2^{\text {Prx1Cre }}$}

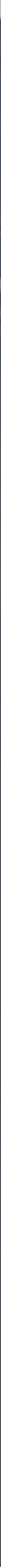

Fig 1 
A.

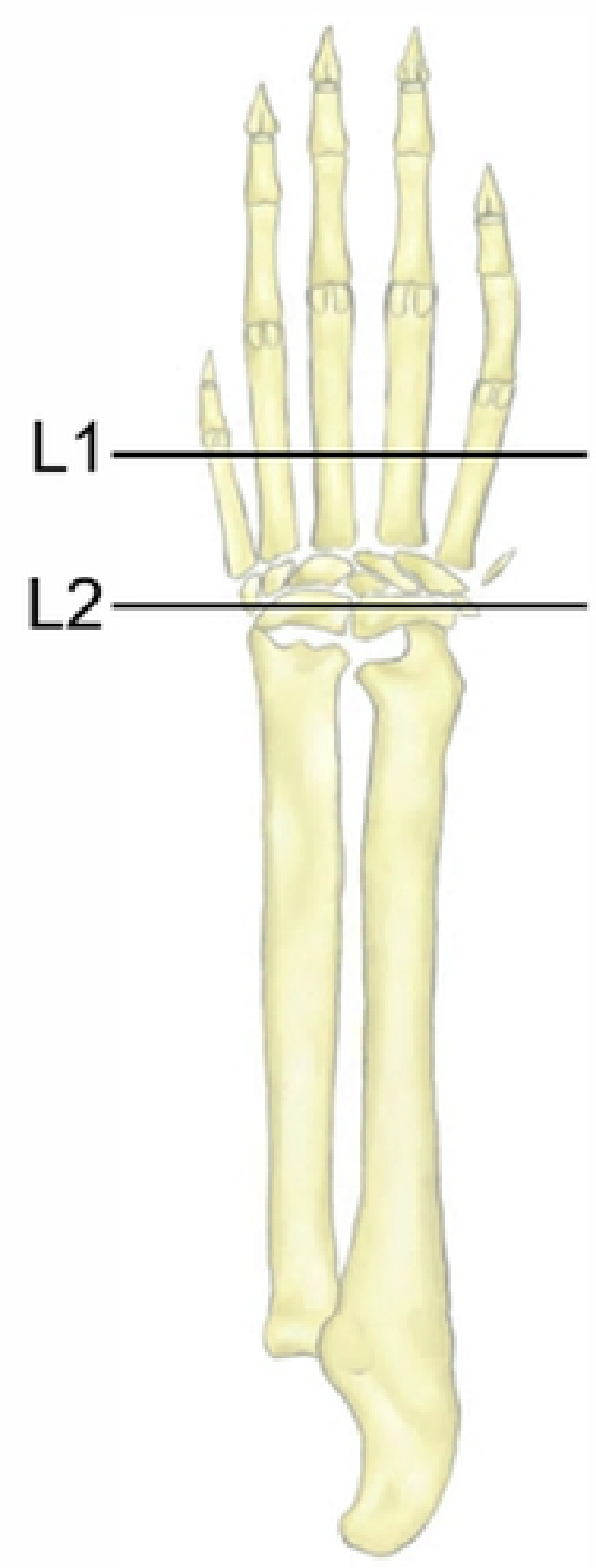

B.

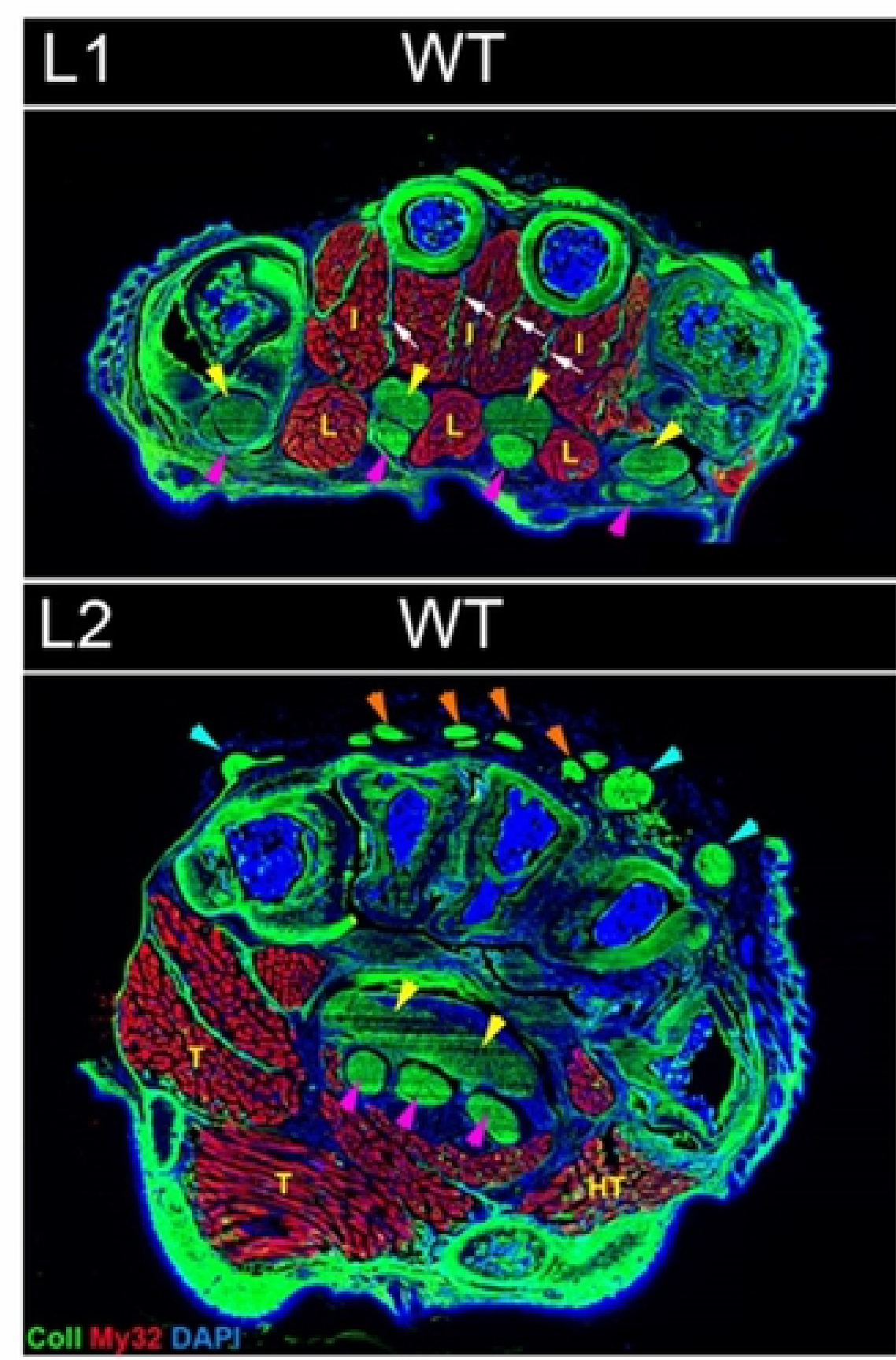

Ezh2 $2^{\text {Prx1Cre }}$

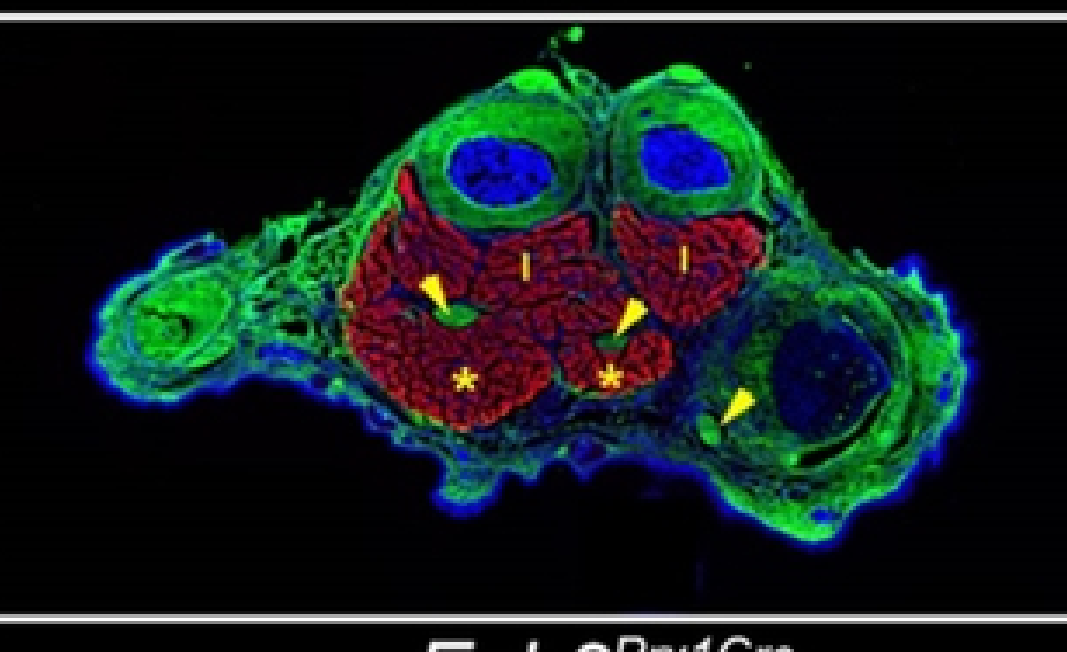

Ezh2Prx1Cre

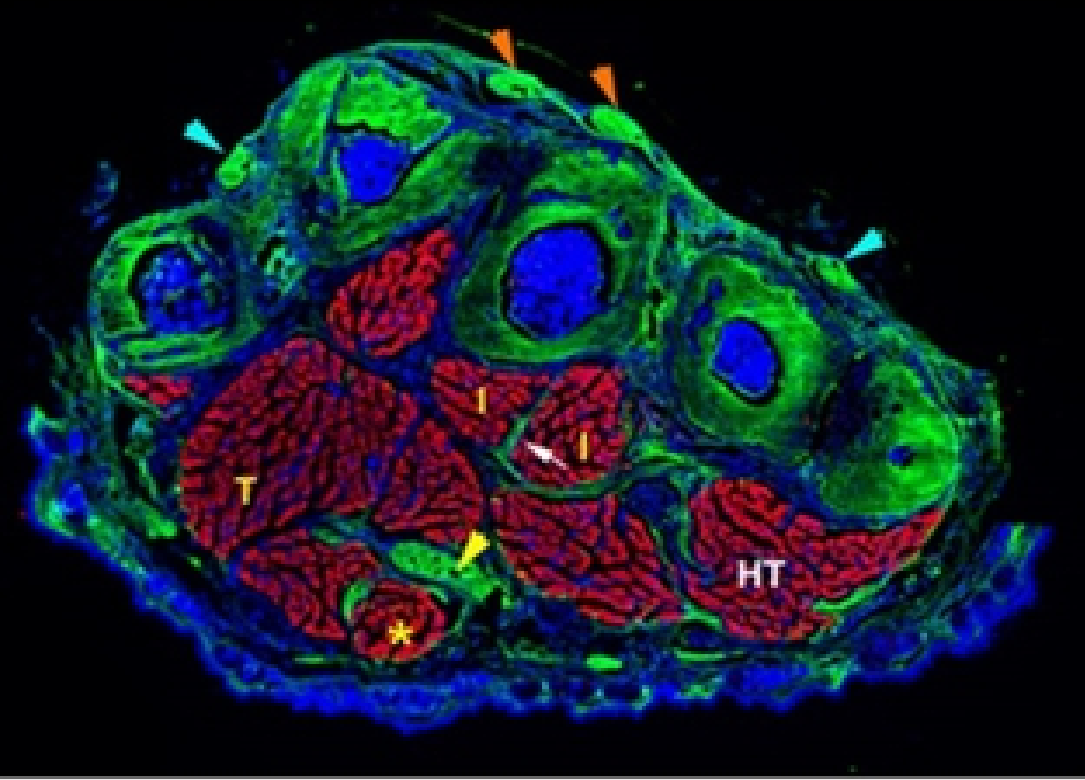

Fig 2 
A.

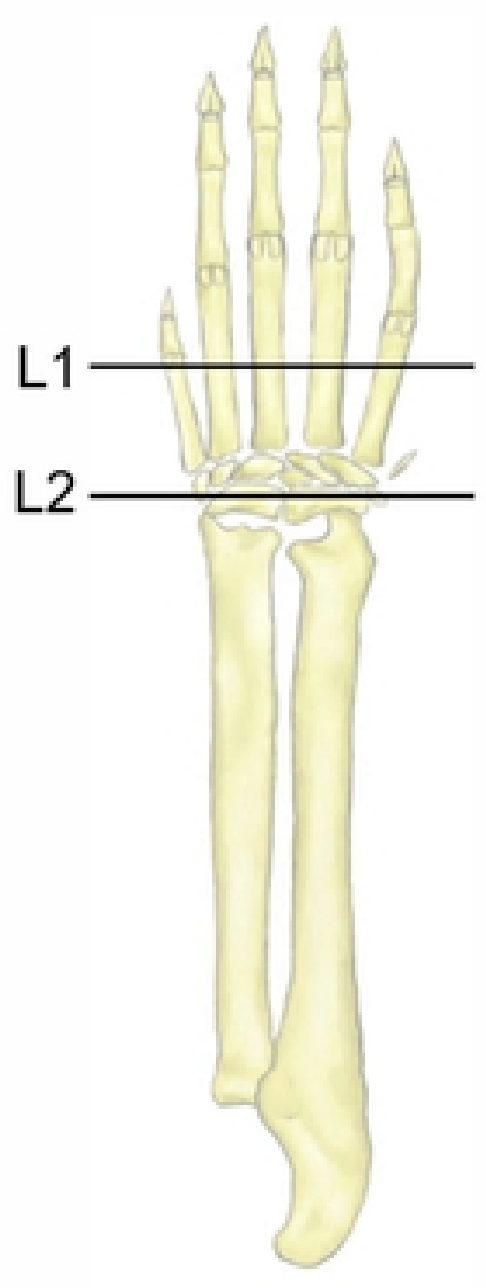

B.
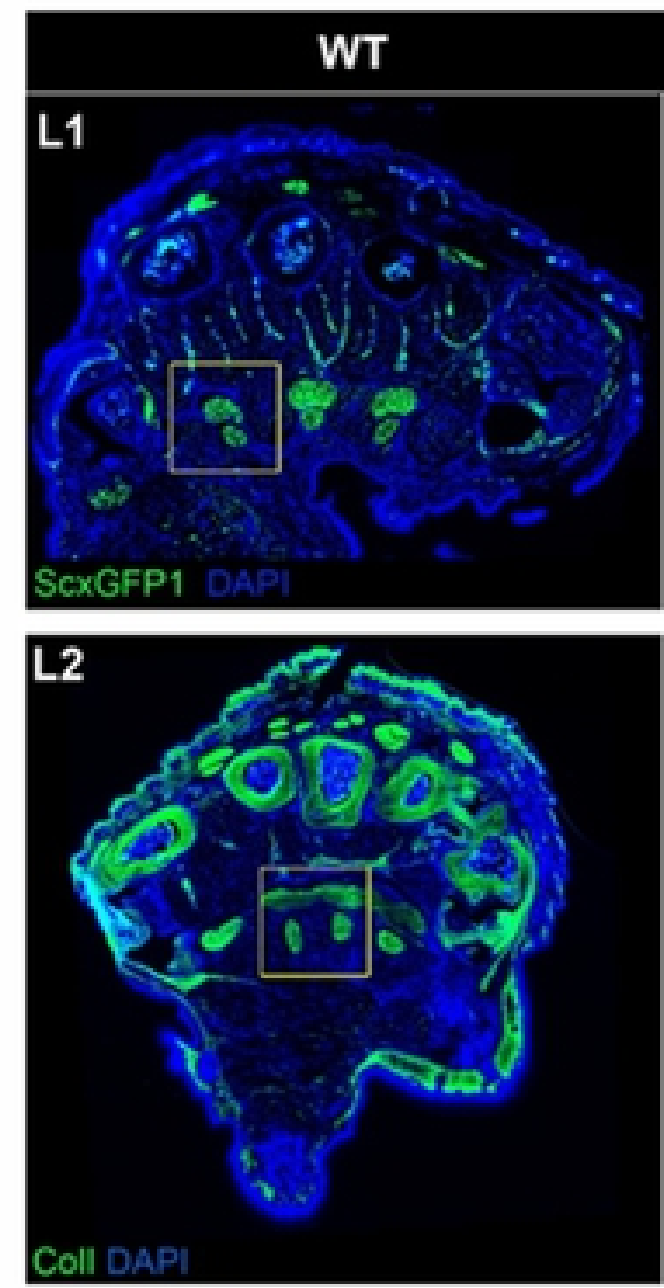

C.
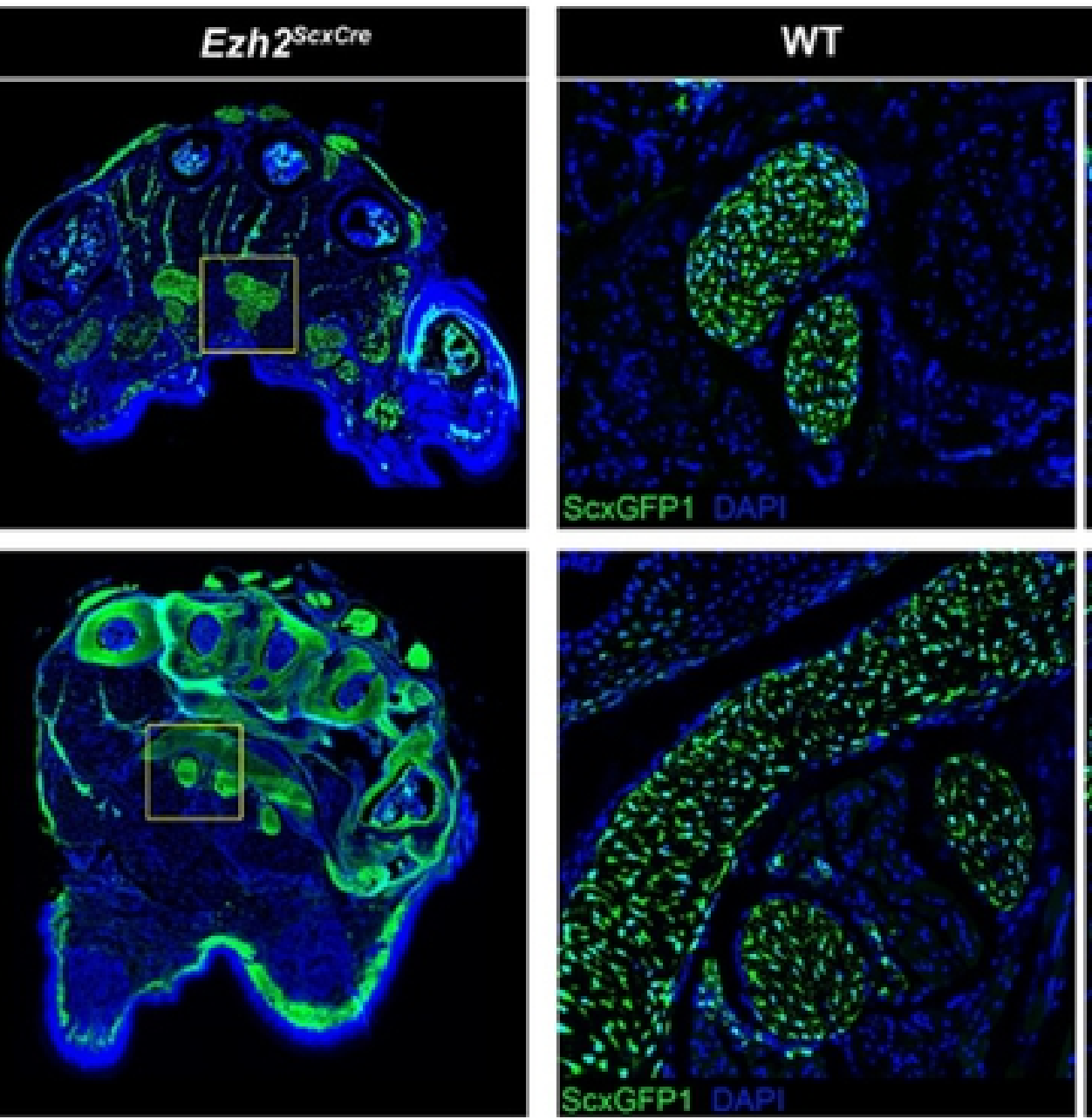

\section{Ezh2 $2^{\text {Sexcre }}$}
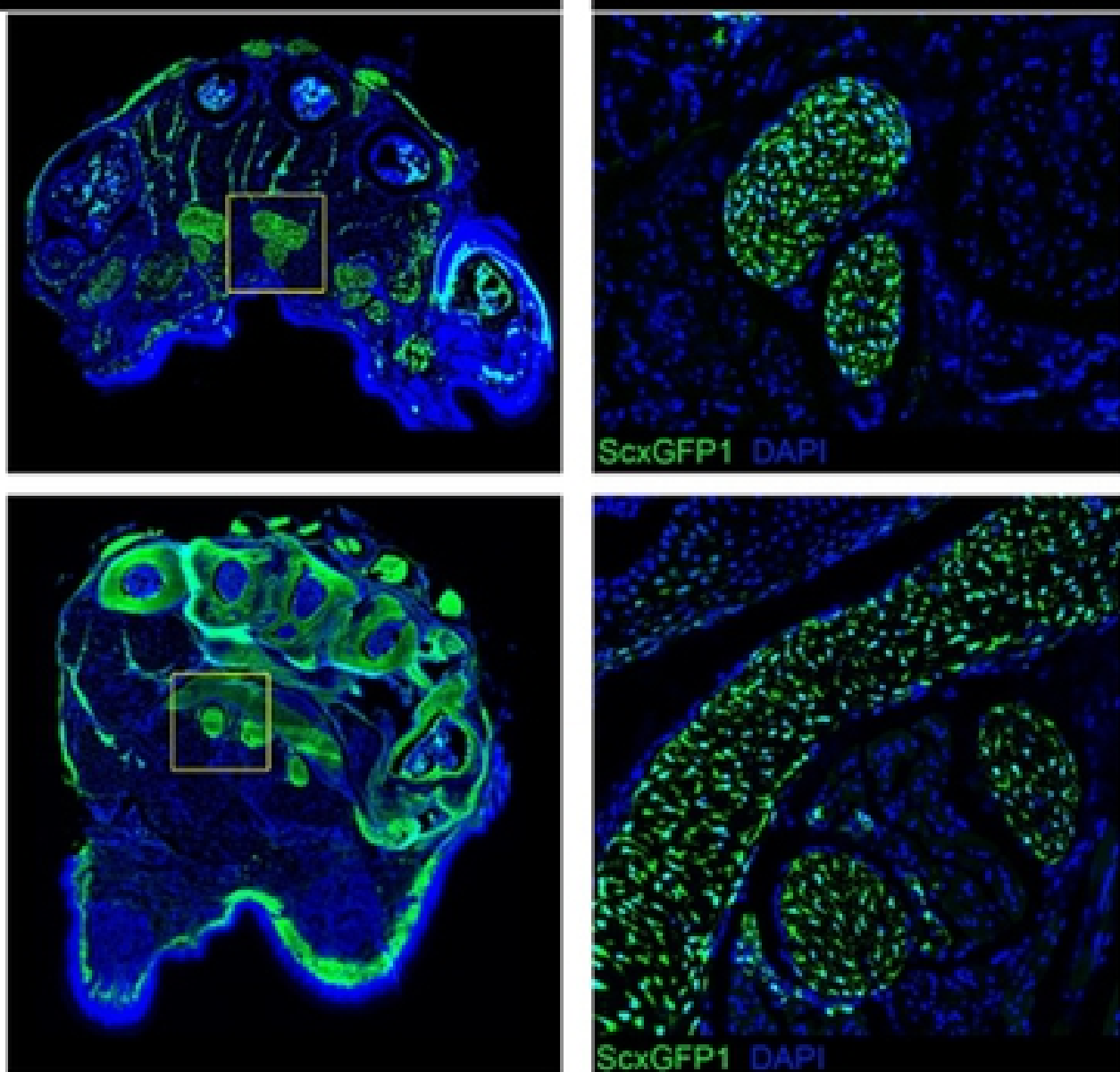

\section{Ezh2 ${ }^{\text {Sexcre }}$}
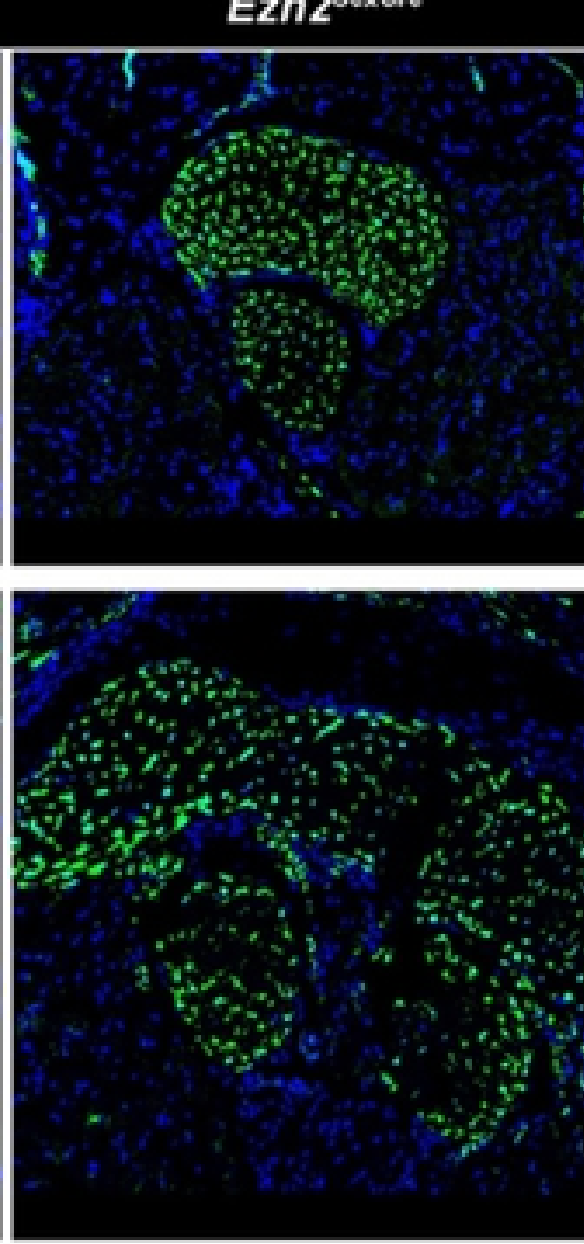

Fig 4 
A.

B.

\section{A.}

L1

WT

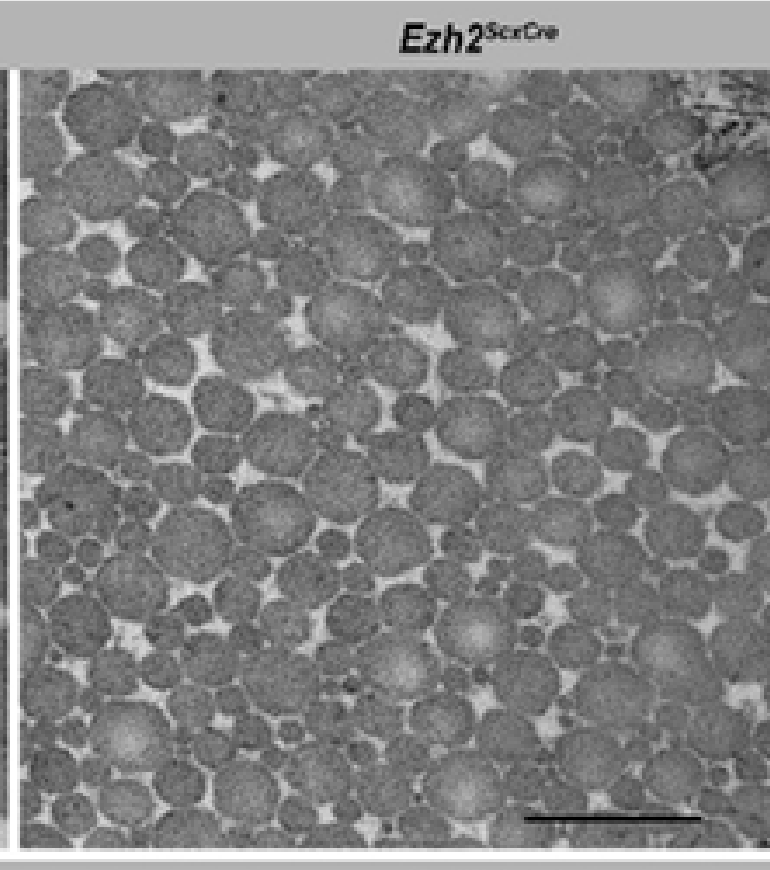

WT

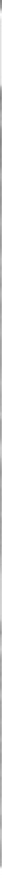

WT

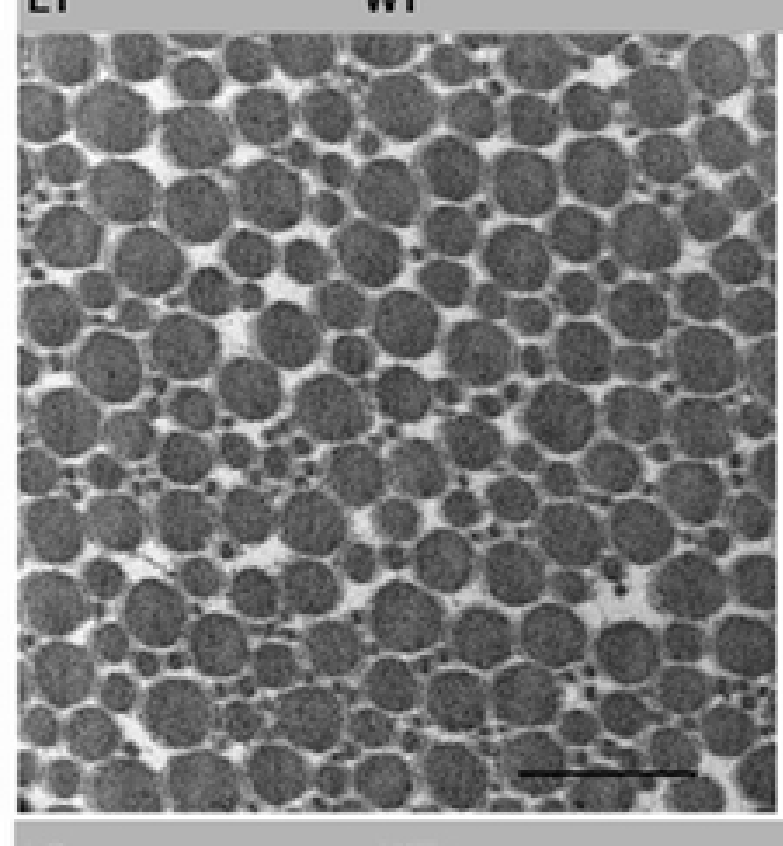

\section{wT}

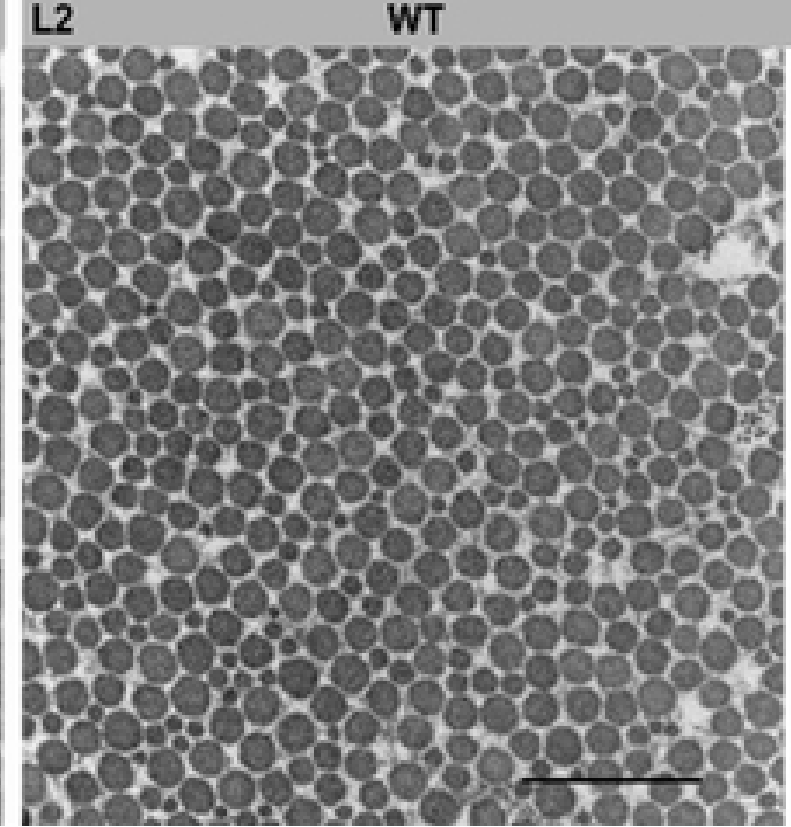

Ezh2

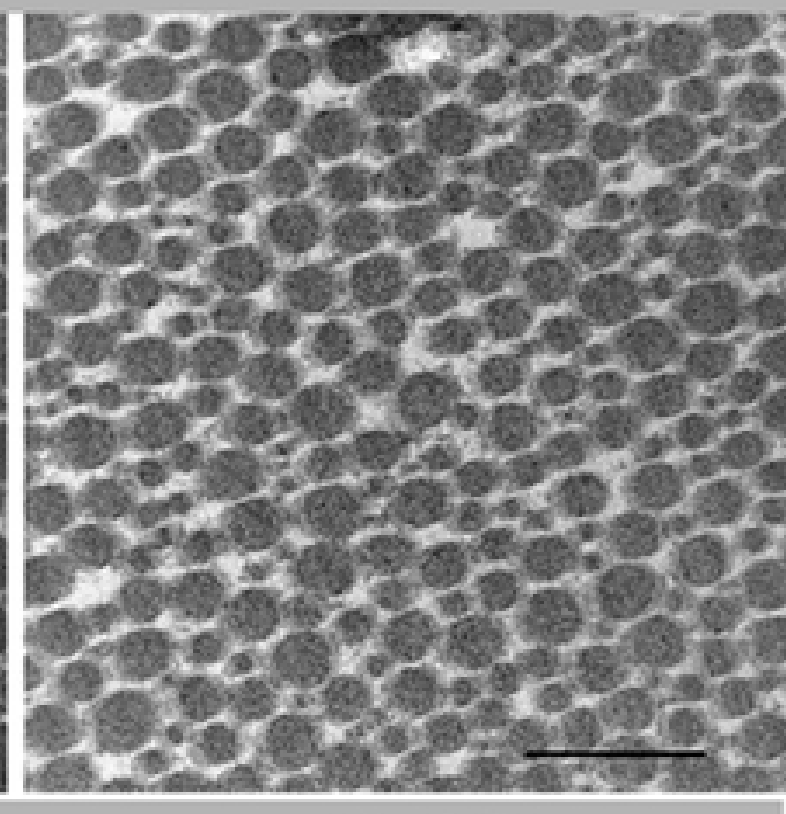

Ezh2 $2^{\text {extaro }}$

Fig 5 